\title{
La tecnología móvil como herramienta de apoyo en la educación media
}

Jorge Armando Aparicio'

Carlos Antonio Aguirre ${ }^{2}$

Edwin Alberto Callejas ${ }^{3}$

Recibido: 15/06/2013 - Aceptado: 11/09/2013

\section{Resumen}

La investigación tuvo por objetivo el desarrollo de una aplicación móvil que apoye el proceso de enseñanzaaprendizaje en la educación media. Se realizó en dos etapas: la primera consistió en hacer un estudio exploratorio descriptivo, en el cual se pasaron encuestas a estudiantes de este nivel para identificar si contaban con dispositivos móviles, el uso que les dan y si estos pueden utilizarse como medio de retroalimentación de sus clases. A partir de los resultados obtenidos se desarrolló una aplicación móvil que permita utilizar un dispositivo móvil para retroalimentar el contenido curricular de educación media. La asignatura que se tomó como base para el desarrollo de la aplicación móvil fue Informática.

\section{Palabras clave}

Aprendizaje-aparatos e instrumentos-investigaciones, máquinas de enseñar, enseñanza con ayuda de computadores, educación tecnológica, medios de enseñanza, sistemas de almacenamiento y recuperación de Información, tecnología móvil.

\section{Abstract}

The research presented here aims to develop a mobile application to support the teaching-learning process in secondary education. It was performed in two stages: The first was to make an exploratory descriptive study, which surveys students passed this level to identify whether they have mobile devices how they use them and if they can be used as a means of feedback for their classes. From the results we developed a mobile application that allows the use of a mobile device for feeding the curricular content of education. The development of the mobile application was conducted on the Computer subject course.

\section{Keywords}

Learning-devices \& instruments-research, teaching machines, computer-assisted education, technology education, educational media, storage systems and information retrieval, mobile technology.

\section{Introducción}

Los rápidos avances tecnológicos, el surgimiento de dispositivos que facilitan la movilidad de la información y comunicación, así como los cambios de paradigmas en las sociedades permiten descubrir alternativas innovadoras, que ayudan a eliminar barreras que impiden el desarrollo

académico y el conocimiento en la población, creando nuevos ambientes de aprendizaje que son el fruto del aprovechamiento tecnológico de esta nueva era.

En la presente investigación se hace un estudio, con estudiantes de educación media para identificar en ellos el tipo de dispositivo móvil que poseen, así como el uso que

1 Jorge Armando Aparicio. Ingeniero Electricista. Director de la Escuela de Informática, Universidad Tecnológica de El Salvador. jorge.aparicio@utec.edu.sv.

2 Carlos Antonio Aguirre Ayala. Licenciado en Administración de Empresas con especialidad en Computación. Encargado del Centro de Investigación de Tecnología Móvil, Universidad Tecnológica de El Salvador. carlos.aguirre@utec.edu.sv.

3 Edwin Alberto Callejas. Ingeniero en Sistemas Informáticos. Docente Tiempo Completo. Escuela de Informática, Universidad Tecnológica de El Salvador edwin.callejas@utec.edu.sv. 
le dan, para así desarrollar una aplicación que motive a los estudiantes a utilizar sus dispositivos móviles para retroalimentar las clases. El trabajo describe las bases teóricas, la metodología de investigación, así como los resultados obtenidos en esta. Posteriormente se presentan las conclusiones, que son la base fundamental para el desarrollo de la aplicación móvil por medio de la cual se podrá apoyar el proceso enseñanza-aprendizaje.

\section{Objetivos}

Con esta investigación se pretende:

a. Identificar el tipo de dispositivos móviles que tienen los estudiantes de educación media para poder así tener la base para la selección del tipo de dispositivo móvil para el cual se desarrollará la aplicación.

b. Obtener el listado de los sistemas operativos que utilizan los dispositivos móviles que tienen los estudiantes de educación media para poder determinar el tipo de sistema operativo que servirá de plataforma base para desarrollar la aplicación móvil.

c. Identificar entre los estudiantes de educación media cuántos de ellos conectan sus dispositivos móviles a internet, ya sea de forma parcial o permanente. Con esto se podrá ver si la aplicación podrá ser descargada por parte de estos desde la web.

d. Desarrollar una aplicación móvil que apoye el proceso de enseñanza-aprendizaje en la educación media.

\section{Marco teórico}

\section{La tecnología móvil, para el desarrollo del conocimiento en la sociedad}

Los avances en la ciencia y la tecnología han traído consigo un cambio sustancial en las prácticas de todas las esferas de la sociedad. Tal es el caso de la educación, donde ahora los procesos de enseñanza-aprendizaje pueden ser guiados por recursos didácticos que tienen como herramienta base el uso de tecnologías. La implantación de estos medios ha generado el diseño de diversos ambientes, más allá de los presenciales, por lo que ahora es común que se hable de ambientes e-learning y m-learning (Montoya, 2009).
El e-learning es un proceso de enseñanza-aprendizaje a través de una computadora, orientado a adquirir competencias por parte del estudiante. Se caracteriza por el uso de la tecnología web, la interacción con la red de estudiantes, tutores y una serie de mecanismos adecuados de evaluación. Este conjunto de servicios de valor agregado puede ayudar a lograr la máxima interacción entre profesor y alumno, garantizando de esta forma la más alta calidad en el proceso de enseñanza-aprendizaje (López, 2010).

Se identifican cuatro etapas en la historia del e-learning: antes de 1983 , cuando no se utilizaban computadoras, solamente métodos tradicionales; 1984-1993, cuando los cursos se entregaban en CD-ROM; 1984-1999, que fue el auge del correo electrónico, donde ahora el maestro se puede comunicar con el alumno por esta vía; y finalmente 2000-2005, cuando la nueva tecnología convierte al e-learning en lo que actualmente conocemos (López, 2010).

El e-learning es elegido por las empresas para capacitar a sus empleados, más que el método tradicional de lectura. Esto debido a que, en esta sociedad globalizada, los empleados tienden a viajar mucho y a movilizarse constantemente de lugar. Además, el e-learning representa un ahorro en costos de capacitación y mejor aceptación por parte de los empleados (López, 2010).

El e-learning ha tenido éxito. Sin embargo, ha llegado a un punto donde necesita evolucionar y madurar. De aquí surge el aprendizaje móvil, permitiendo nuevas posibilidades, integrando nuevas tecnologías y calidad en la educación. Puede definirse que el aprendizaje móvil es la capacidad de usar la tecnología móvil para lograr una experiencia de aprendizaje en el alumno. Este tipo de aprendizaje se puede utilizar en diversos ámbitos, no solo en la escuela. Puede utilizarse en las empresas para capacitar a los empleados, para hacer labor de campo y para realizar campañas promocionales. El aprendizaje móvil promete apoyar la enseñanza virtual por medio de nuevos dispositivos portátiles, que fomenten nuevos sistemas de comunicación entre el hombre y la tecnología (López, 2010).

Son dispositivos móviles: tablets, iPod, Smartphone, y otros, los cuales permiten compartir material auditivo, vídeo, tex- 
to, imágenes y archivos con los cuales se facilita el proceso de enseñanza-aprendizaje (Caffa, 2008).

Para los estudiantes, las herramientas de aprendizaje móvil resultan más atractivas, ya que logran interactuar con ellas todo el tiempo. La gran ventaja de estos dispositivos es que el estudiante puede llevarlos consigo a donde vaya, así como puede ayudar a eliminar un poco la formalidad que existe en un método de aprendizaje tradicional, siendo esto más cómodo y amigable para los estudiantes, sobre todo para los jóvenes que buscan siempre la oportunidad de aprender en materia tecnológica, además, ayuda a combatir la resistencia al cambio tecnológico que los adultos tienen (López, 2010).

Algunos recursos y aplicaciones que se encuentran disponibles y factibles de ser integrados en los ambientes de aprendizaje son los siguientes: blogs, sistemas de administración de cursos, mensajes instantáneos, wikis, podcast, RSS, espacios sociales y otras herramientas de la web. Estos recursos tecnológicos están siendo integrados en los ambientes de aprendizaje a distancia, multimodales, combinados o de m-learning. El uso y las posibilidades que pueda hacerse con ellos están en relación directa con los aprendizajes que se quiera promover, y, por ello, la creatividad en el diseño juega un papel importante, así como las condiciones de implan- tación que se realicen para que sean integrados en estos ambientes (López, 2010).

\section{Metodología}

La investigación se realizó con el objetivo de identificar el tipo de dispositivo móvil que los estudiantes poseen, así como el uso que estos les dan. Se hizo un estudio exploratorio descriptivo por medio del cual se identificaron los datos necesarios para desarrollar la aplicación. Con esta información se desarrolló la aplicación móvil que podrá ser accedida e instalada por los estudiantes en sus dispositivos móviles, convirtiendo a este dispositivo en un apoyo al proceso de enseñanza-aprendizaje en la educación media.

\section{Participantes}

El proceso de recolección de datos se realizó en centros de estudio de educación media en el departamento de San Salvador, en la zona metropolitana del municipio de San Salvador. Se seleccionaron aleatoriamente catorce centros de estudio; de cada uno se encuestaron veinte estudiantes de entre 15 a 22 años, teniendo un total de 280 encuestados. A continuación se presenta el listado de las instituciones encuestadas.

Tabla 1. Alumnos encuestados

\begin{tabular}{lcc}
\multicolumn{1}{c}{ Institución } & Tipo de institución & MUESTRA \\
\hline Liceo Cristiano Reverendo Juan Bueno Central & Colegio & 20 \\
Centro Educativo Joya de Cerén & Colegio & 20 \\
Queens School & Colegio & 20 \\
Colegio Cristóbal Colón & Colegio & 20 \\
Colegio Eucarístico & Colegio & 20 \\
Colegio La Asunción & Colegio & 20 \\
Colegio Guadalupano & Colegio & 20 \\
Instituto Nacional Francisco Morazán & Instituto & 20 \\
Instituto Técnico Exsal & Instituto & 20 \\
Instituto Nacional Técnico Industrial & Instituto & 20 \\
Instituto Técnico Ricaldone & Instituto & 20 \\
Instituto Nacional Albert Camus & Instituto & 20 \\
Instituto Politécnico Nazareth & Instituto & 20 \\
Instituto Nacional Alberto Masferrer & Instituto & 20 \\
\hline
\end{tabular}




\section{Instrumento}

El cuestionario busca identificar el tipo de dispositivos móviles que los estudiantes de educación media tienen; cuáles son los usos que estos le dan al dispositivo; el tipo de sis- tema operativo móvil; si ellos ya han tenido experiencia en instalación de aplicaciones en sus dispositivos, entre otros aspectos que se describirán a lo largo del documento. La encuesta se pasó en el año 2011. Las preguntas se enumeran a continuación.

Tabla 2. Preguntas encuesta

\begin{tabular}{ll} 
No. & \multicolumn{1}{c}{ Preguntas } \\
\hline 1 & ¿Qué entiende por dispositivo móvil? \\
3 & De los dispositivos móviles que a continuación se listan, ¿cuál es el que posee? \\
4 & De las marcas de dispositivos móviles que se muestran, ¿a cuál pertenece su dispositivo móvil? \\
5 & De las siguientes definiciones, ¿cuál considera que define el término SO? \\
6 & De los nombres de SO que se listan, ¿cuál es el que más ha escuchado mencionar? \\
7 & ¿Qué tipo de So tiene su dispositivo móvil? \\
8 & De los siguientes rangos de precio, ¿cuánto pagaría por un dispositivo móvil? \\
9 & De la siguiente lista de móviles, ¿cuál compraría? \\
10 & ¿Para qué utiliza el dispositivo móvil? \\
11 & ¿Su dispositivo móvil tiene conectividad a internet? \\
13 & De los siguientes So para móviles, ¿cuál considera que es más flexible con el usuario? \\
14 & ¿Ya ha instalado aplicaciones en un dispositivo móvil? \\
15 & Del siguiente listado, ¿qué tipo de aplicaciones ha instalado? \\
16 & ¿Utilizaría su dispositivo móvil para retroalimentar sus clases en la educación media? \\
& Seleccione uno o varios del tipo de aplicación con la cual le gustaría retroalimentar su clase. \\
\end{tabular}

\section{Procedimiento}

Lo primero que se hizo fue ir a los centros de estudio a pasar la encuesta. Este proceso se llevó a cabo en la segunda semana de julio del año 2011, en los centros de estudio de educación media de la zona metropolitana de San Salvador.

Posteriormente, se hace el procesamiento de la información recolectada. Este proceso fue muy significativo, se realizó entre el mes de julio y agosto del 2011, por medio del cual se obtienen resultados que permiten hacer un análisis con el que se establecen conclusiones que describen información para el desarrollo de la aplicación móvil.
Cuando ya se tienen los resultados y el análisis de la información, ahora se cuenta con las bases para desarrollar la aplicación que permita utilizar los dispositivos móviles como herramienta de apoyo en la educación media. En el año 2012, entre los meses de marzo y mayo, se obtiene el plan de estudio de la asignatura de Informática para educación media; con base a este plan se desarrolló un test que incluyó todos los contenidos de la asignatura. Se finalizó la aplicación en el mes de octubre de 2012. 


\section{Resultados}

A continuación se presentan los resultados obtenidos de la encuesta.

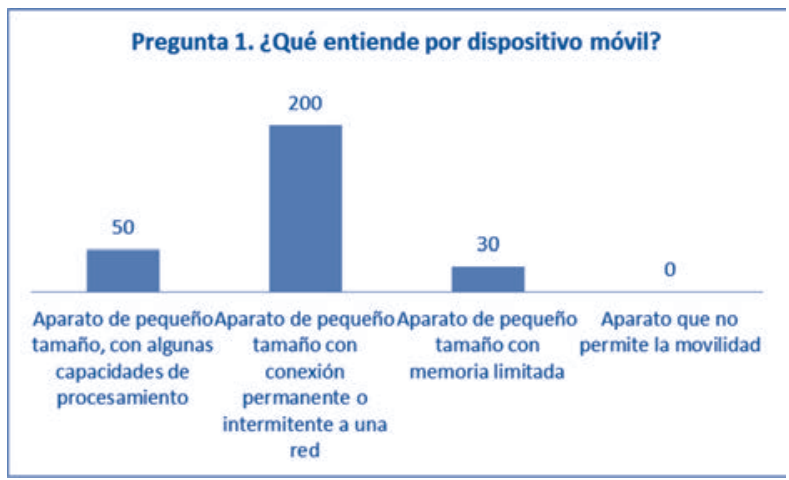

Figura 1. Muestra que todos los encuestados pueden definir qué es un dispositivo móvil. Esto permite garantizar que tienen claro el concepto.

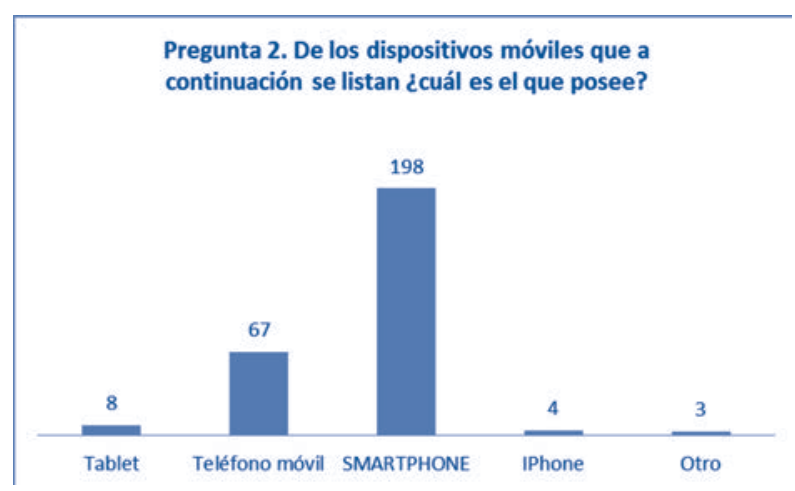

Figura 2. Muestra que los smartphones son los más populares entre los estudiantes encuestados, lo que permite abrir amplias posibilidades para la utilización de los dispositivos móviles en la educación media.

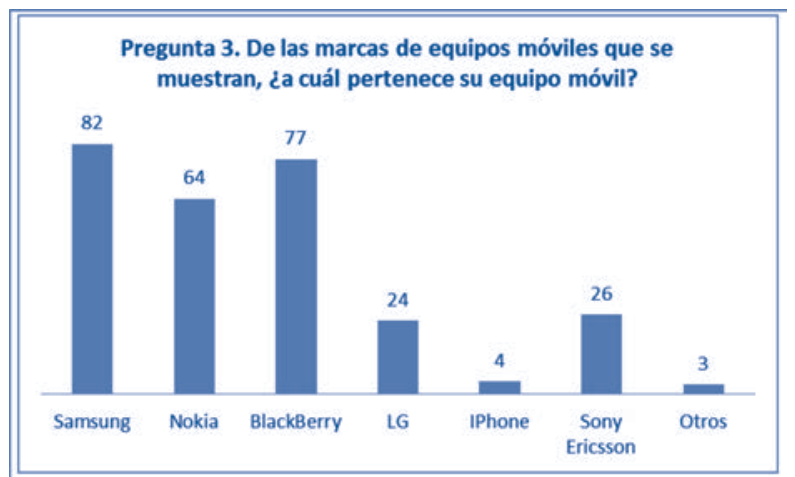

Figura 3. Las marcas más posicionadas en los encuestados, en su orden, son Samsung, BlackBerry, Nokia, LG, Sony Ericsson.

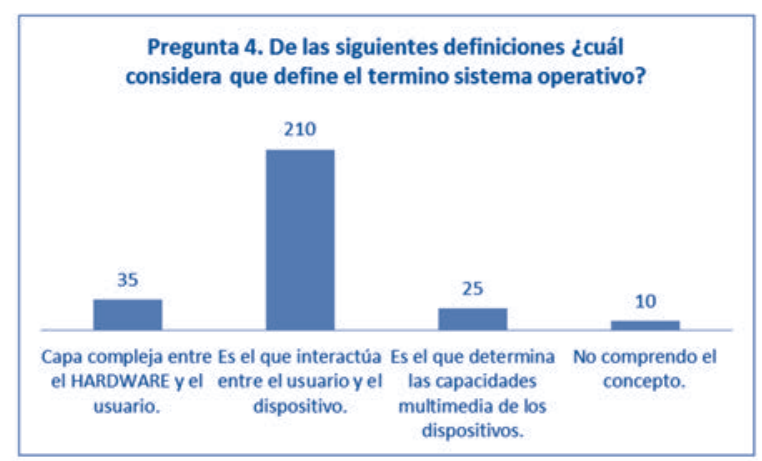

Figura 4. La mayor parte de encuestados sabe definir que es un sistema operativo. Lo que permite tener libertad al momento de desarrollar la aplicación móvil. 


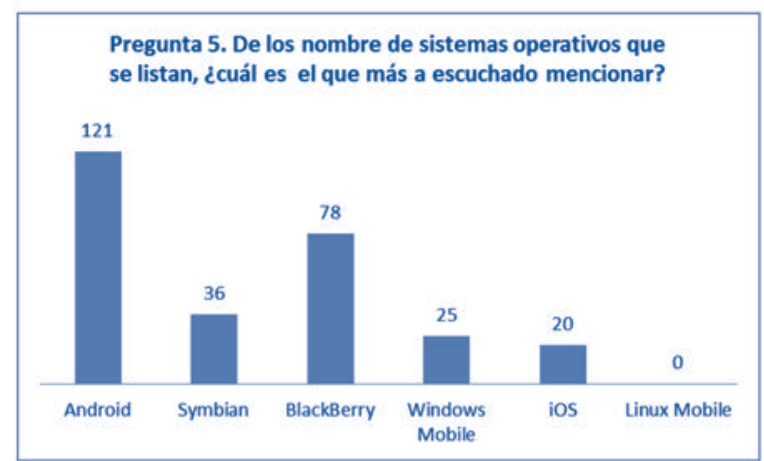

Figura 5. Muestra que el sistema operativo para móviles más conocido entre los estudiantes encuestados es el sistema Android, le siguen BlackBerry, Symbian, Windows Mobile e iOs.

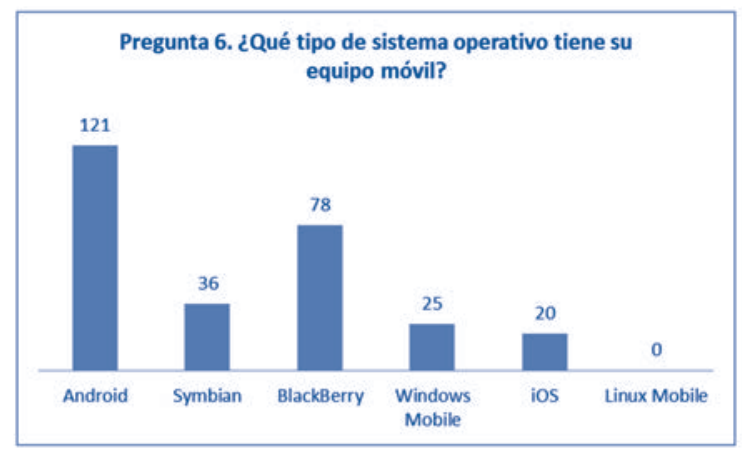

Figura 6. Muestra que el sistema operativo que más tienen los encuestados es el Android.

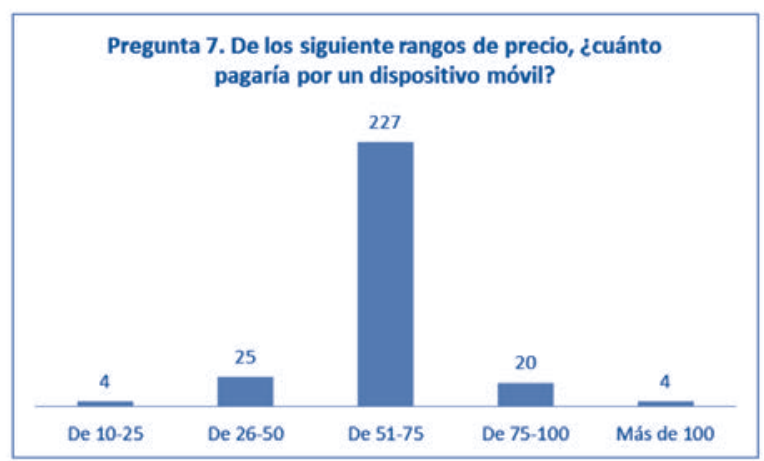

Figura 7. Muestra que la mayoría de los estudiantes estarían dispuestos a pagar entre 51 y 75 dólares por un dispositivo móvil. Con esto se tiene un parámetro que se debe considerar al momento de desarrollar la aplicación.

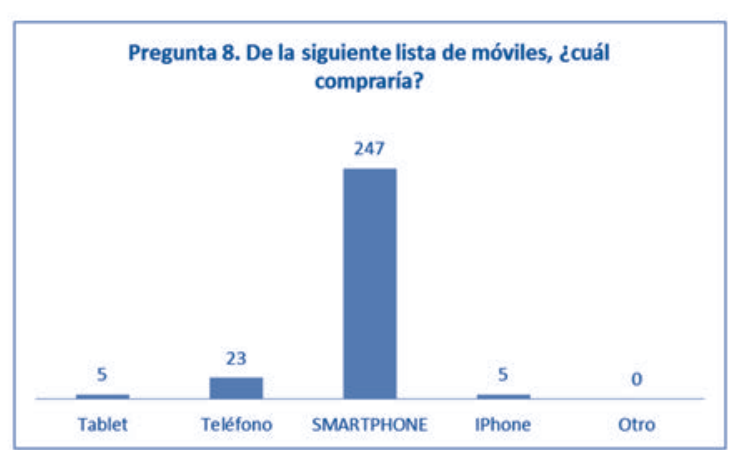

Figura 8. La mayor parte de estudiantes preferirían comprar smartphones. Esto es indicador a favor, ya que los smartphones reúnen las características necesarias para el aprendizaje móvil.

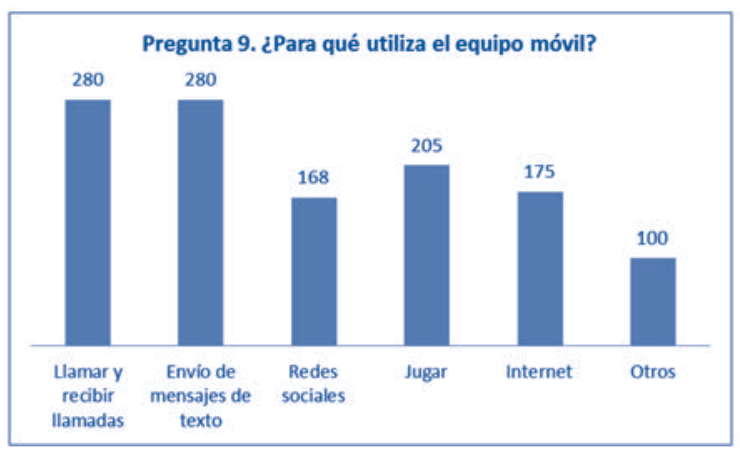

Figura 9. Muestra que la mayor parte de estudiantes utiliza el teléfono para envío y recepción mensajes, así como para recibir y hacer llamadas. Un número significativo utiliza internet, redes sociales y juegos en su dispositivo móvil.

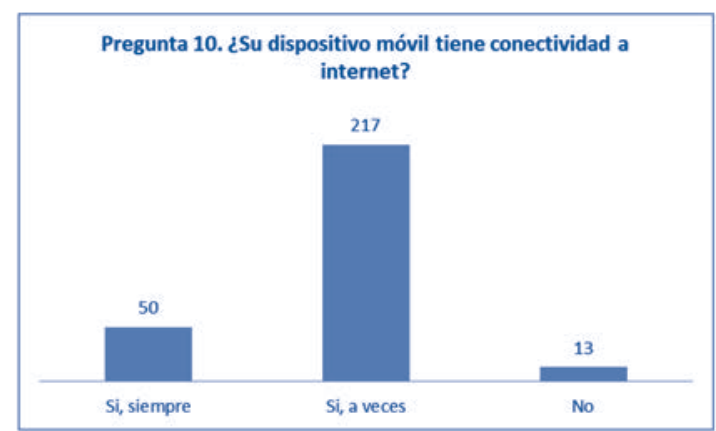

Figura 10. Muestra que la mayoría de los estudiantes encuestados tienen en sus dispositivos móviles conectividad a internet. 


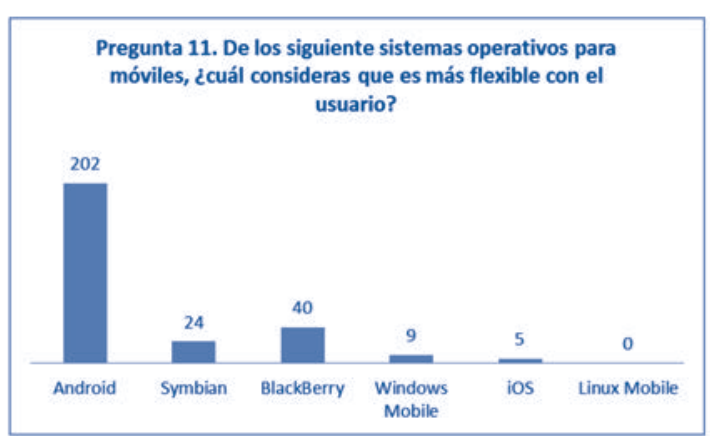

Figura 11. Muestra que los estudiantes encuestados consideran que el sistema operativo más flexible es Android.

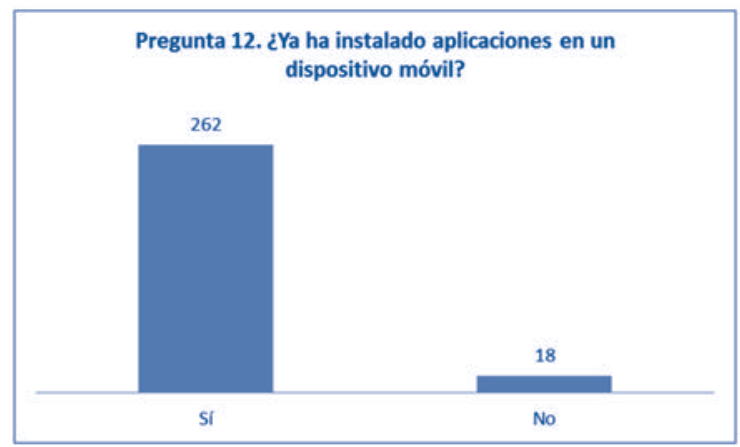

Figura 12. Muestra que la mayor parte de los estudiantes ya ha instalado aplicaciones en sus dispositivos móviles.

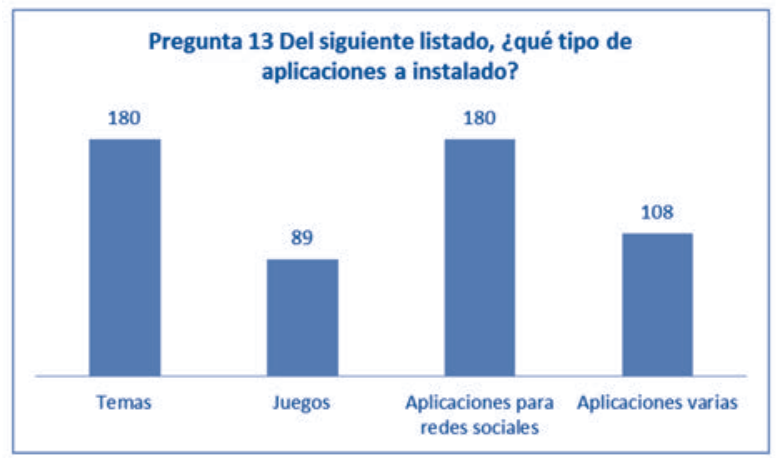

Figura 13. Las aplicaciones que más instalan los estudiantes en sus dispositivos móviles están relacionadas con las redes sociales, los juegos y otras.

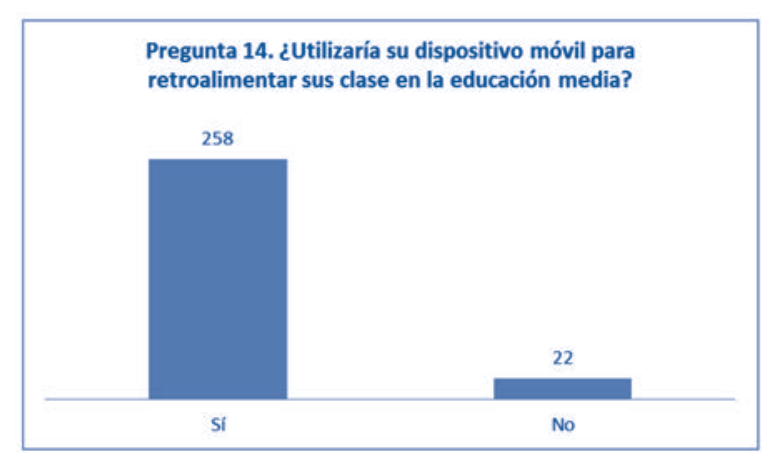

Figura 14. La mayor parte de los estudiantes encuestados estaría de acuerdo con utilizar su dispositivo móvil para retroalimentar sus clases.

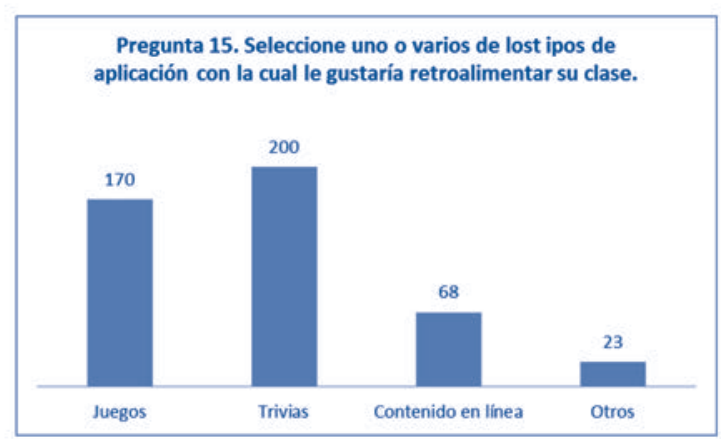

Figura 15. A la mayor parte de estudiantes encuestados les gustaría retroalimentar sus clases por medio de trivias o juegos.

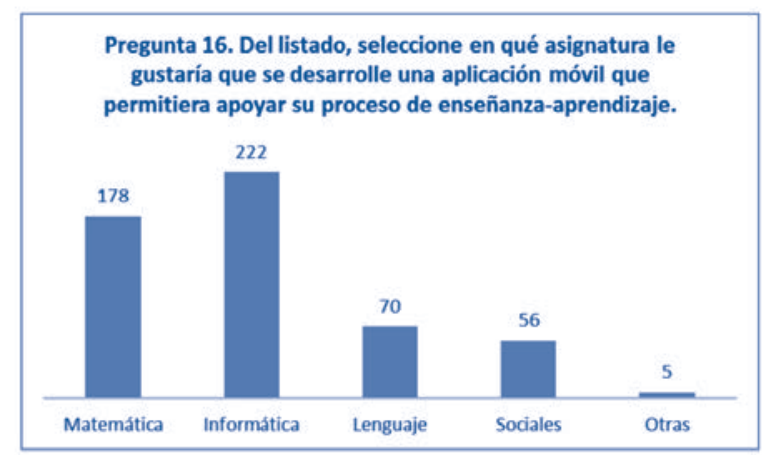

Figura 16. A la mayor parte de los estudiantes les gustaría recibir clases de informática utilizando sus dispositivos móviles. 


\section{Discusión de resultados}

Después de presentar los resultados a través de la encuesta, se hace un análisis de los aspectos que son la base fundamental para el desarrollo de la aplicación móvil, que se convierte en el producto final de esta investigación. Por lo que se concluye lo siguiente:

Los smartphones son los dispositivos móviles más populares entre los estudiantes de educación media. Con esto se amplía la posibilidad de utilizar un dispositivo móvil como herramienta de apoyo al proceso enseñanza-aprendizaje.

Las marcas de dispositivos móviles más posicionadas en los estudiantes de educación media son, en su orden, Samsung, BlackBerry, LG, Sony Ericsson, y otros.

El sistema operativo más utilizado en los dispositivos móviles de los estudiantes de educación media es Android, considerando que es flexible, amigable y sobre todo es de ambiente libre. Con esta información se parte como base para la creación de la aplicación móvil, la cual se desarrolló para el ambiente Android. Adaptando el sistema a dispositivos no tan sofisticados ni con altos costos, ya que la mayor parte de estudiantes estarían dispuestos a pagar por un móvil entre 51 a 75 dólares. Otro dato interesante es que, ante la diversidad de dispositivos móviles existentes, los estudiantes preferirían comprar un smartphone.

Los dispositivos móviles que los estudiantes de educación media poseen los conectan a internet, ya sea utilizando un plan de datos dedicado o a través de suscripción promocional. Las aplicaciones que más utilizan son: redes sociales, temas para cambiar apariencia al dispositivo móvil, otras aplicaciones de interés personal. Además de tener ya alguna experiencia en la instalación de aplicaciones móviles. Todo esto abona al proceso de querer implantar m-learning en la educación media; hacer uso de dispositivos móviles como una herramienta para retroalimentar las clases de educación media.
A los estudiantes de educación media les gustaría utilizar su dispositivo móvil para retroalimentar sus clases. La mayoría considera que la asignatura de Informática es la ideal, y entre las aplicaciones que prefieren para aprender señalan las trivias y los juegos. Este aspecto se consideró al momento de desarrollar la aplicación móvil.

\section{Implantación del proyecto}

"Aprendiendo informática por medio de dispositivos móviles con sistema Android"

\section{Diseño del prototipo}

\section{Actividades}

Las actividades son el puente con que el usuario se comunica; tienen cierta capacidad de jerarquía, pues se van ejecutando una tras otra y regresan el control, de tal forma que así como se van abriendo se irán cerrando, regresando el control a la actividad que la llamó.

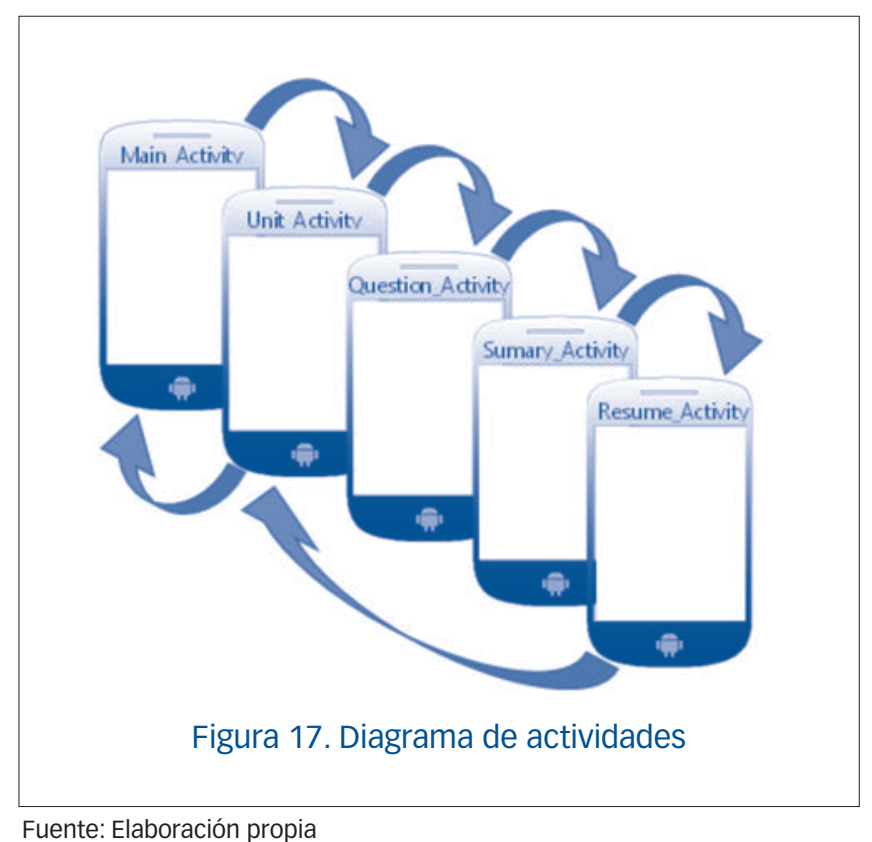

Fuente: Elaboración propia 
Tabla 3. Nombre de actividades y su descripción

Nombre de la actividad

\begin{abstract}
Main
\end{abstract}
Unit

Question

Summary

Resume

About

\section{Descripción}

Actividad de entrada de la aplicación.

Lista de unidades.

Manejo de preguntas.

Estado de la prueba, nota final.

Resumen final, detalle en lo que se falló.

Breve información de la aplicación.

Fuente: Elaboración propia

La tabla anterior muestra los nombres de las actividades que participan en la ejecución de la aplicación.

Código fuente (soporte de las actividades, clases Java)

Se detalla a continuación un listado de las clases Java que dan soporte y control de datos a las actividades que intervienen en la aplicación.

Tabla 4. Clases Java que dan soporte a la aplicación

Actividad

Descripción

\begin{tabular}{|c|c|c|}
\hline $\begin{array}{c}\text { एAVA } \\
\stackrel{(3}{=}\end{array}$ & MainActivity & Código fuente para el manejo de acceso a la aplicación. \\
\hline $\begin{array}{l}\text { UAVA } \\
\stackrel{(S}{=}\end{array}$ & UnitActivity & Listado de unidades que se deben evaluar. \\
\hline $\begin{array}{l}\text { JAVA } \\
\stackrel{(1)}{=}\end{array}$ & QuestionActivity & Control de preguntas aleatorias que se deben mostrar y evaluar. \\
\hline $\begin{array}{l}\text { UAVA } \\
\stackrel{(3)}{=}\end{array}$ & SummaryActivity & Mostrar resultado de aprobado o no aprobado y nota final. \\
\hline $\begin{array}{l}\text { UAVA } \\
\stackrel{(S)}{=}\end{array}$ & ResumeActivity & Detalle de preguntas falladas. \\
\hline $\begin{array}{c}\text { UAVA } \\
\stackrel{(1)}{=}\end{array}$ & AboutActivity & Información de aplicación. \\
\hline 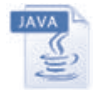 & Matters & Clase constructora para definir objeto de unidades. \\
\hline 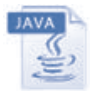 & Questions & Clase constructora para definir objeto de preguntas. \\
\hline $\begin{array}{l}\text { UAVA } \\
\stackrel{S}{=} \\
\stackrel{3}{\Xi}\end{array}$ & Responses & Clase constructora para definir objeto de respuestas. \\
\hline
\end{tabular}




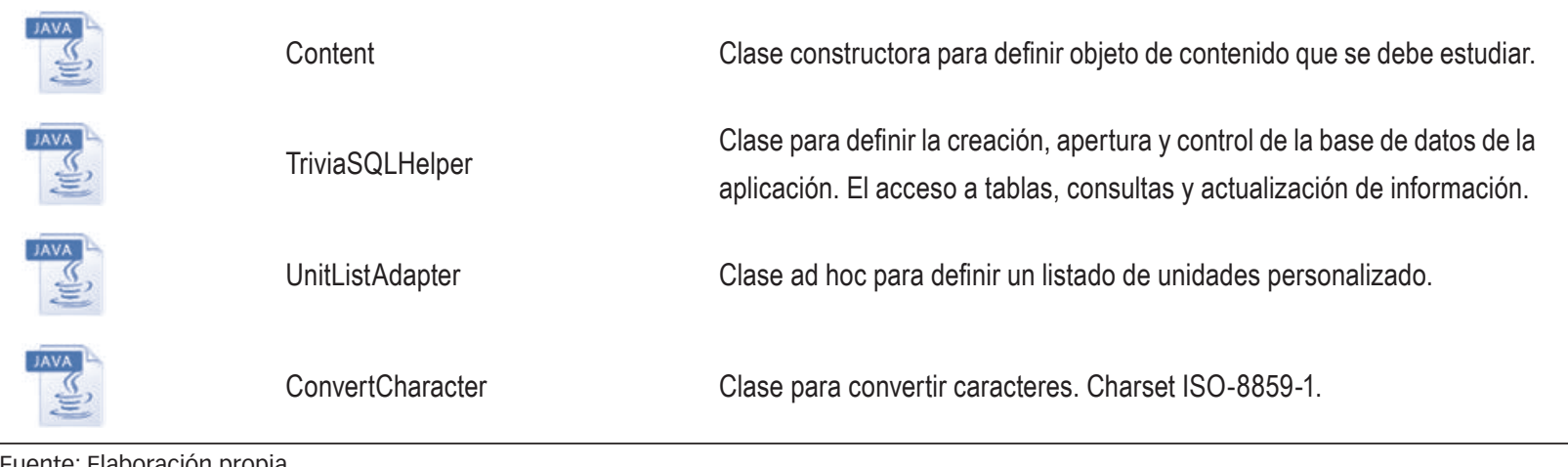

Fuente: Elaboración propia

El anterior listado muestra una descripción general de la función principal de cada clase Java. Sin embargo, el funcionamiento de algunas clases es más amplio. Es necesario, entonces, definir un diagrama que explique de mejor manera cómo cada clase Java se comunica con la otra. Es necesario mostrar el escenario de control de flujo de datos que interactúa entre cada una de ellas. A continuación se presenta en esquema que muestra tal comunicación.

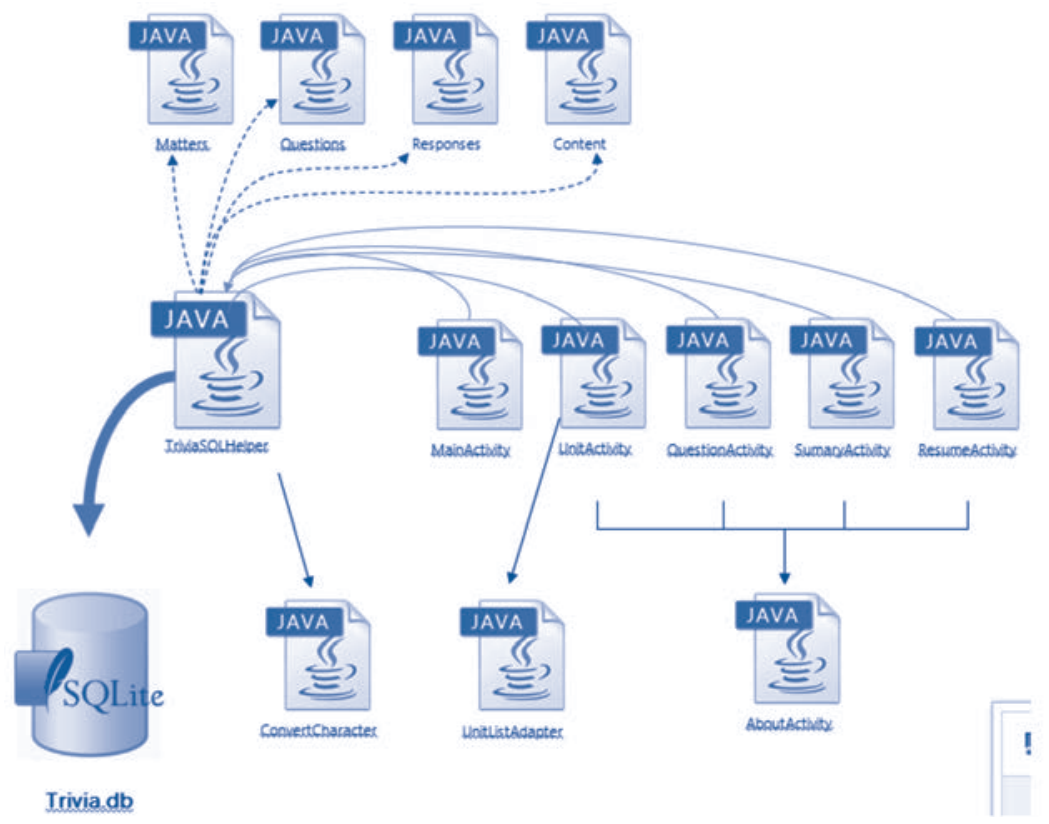

Figura 18. Estructura de trabajo de la aplicación y conexión a la base de datos SQLite

Fuente: Elaboración propia

\section{Base de datos}

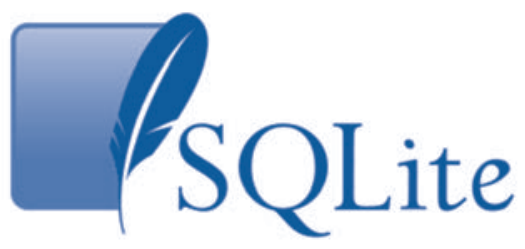

Figura 19. Logo base de datos SQLite

Fuente: Sitio web de Sqlite http://www.sqlite.org 
Se ha utilizado la base de datos por defecto que maneja los sistemas operativos Android, en este caso SQLite, que es una pequeña base de datos capaz de manejar cierta cantidad de información.

La información se compone de unidades, preguntas con sus respectivas respuestas y contenido de estudio. Todo esto conforma las tablas que definen la base de datos Trivia.db.

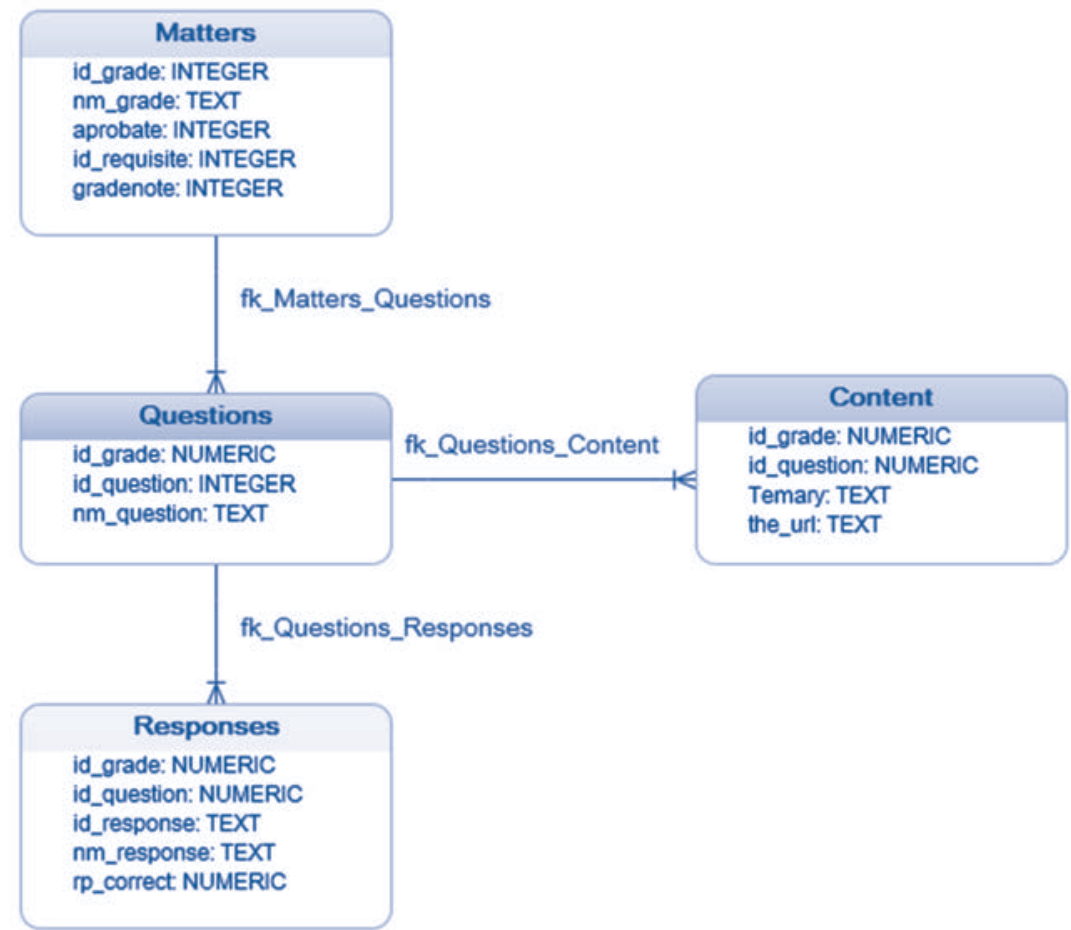

Figura 20. Diseño de la base de datos

Fuente: Elaboración propia

\section{Dificultades que se tuvieron a la hora del di- seño del prototipo}

\section{Contenido estático}

En el diseño actual de la aplicación Trivia para sistemas Android, la aplicación es capaz de ejecutarse tanto en dispositivos móviles que posean un sistema operativo Android 2.3 Gingerbread como en tablets con sistema operativo Android 3 o Honeycomb.

En un principio se detectó el problema de contenido estático; es decir, se encontró que en dispositivos que posean una pantalla de dimensiones pequeñas el contenido quedaba oculto, por lo que se investigó este problema y se obtuvo como solución implantar ScrollViews, que permite tener control de movimiento de contenido, por lo que este problema fue superado.

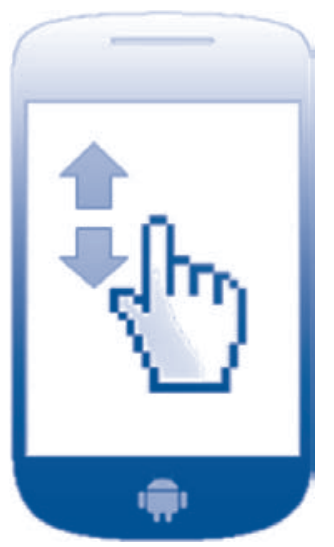

Figura 21.

Fuente: Elaboración propia 


\section{Orientación}

También se detectó un problema mucho más complejo. Este radica en el control de orientación de los dispositivos. En los dispositivos móviles este problema no requiere mucha atención, pues el sistema operativo no reinicia las variables ni componentes de control de datos, ni los de flujo de datos, por lo que, al estar en una orientación vertical y pasarse a una orientación horizontal, los datos que se muestran son los mismos. Este problema lo tienen en su mayoría los sistemas operativos más recientes de las tablets. Al intentar cambiarse entre orientaciones, la información es reiniciada, por lo que se pierde el flujo de control de datos y de la aplicación en sí. Este problema fue detectado al estarse desarrollando la prueba, por ejemplo, en el simulacro de preguntas contestadas. Sí se tenían contestadas dos preguntas de las cinco que se muestran, al cambiar la orientación de la tablet, se volvía a reiniciar el proceso de preguntas; es decir, se iniciaba desde la pregunta 1, quedando el proceso de contestación anterior perdido.

La solución más próxima fue bloquear la orientación de los dispositivos en la actividad de las preguntas. En este caso, la orientación se establece de manera rígida a una orientación vertical, con lo que este problema queda solucionado.

En la actualidad, la aplicación está trabajando de esta manera rígida; pero existe un par de soluciones. En el diseño de la aplicación, en la actualidad, el proceso que genera las preguntas aleatorias se encuentra ubicado en la actividad QuestionActivity, con lo que cada vez que se cambia de orientación este proceso es reiniciado.

La primera solución sería la de manejar una variable global que controle, en esta actividad, tal proceso; que dé paso a la generación del proceso una sola vez. Otra solución sería la de reubicar el proceso en una actividad anterior, en la UnitActivity. Esta actividad es la encargada de mostrar las unidades en evaluación. Cuando el usuario desee entrar en alguna de esas actividades, se debe lanzar el proceso de creación de las preguntas aleatorias, para que la siguiente actividad solo las reciba.

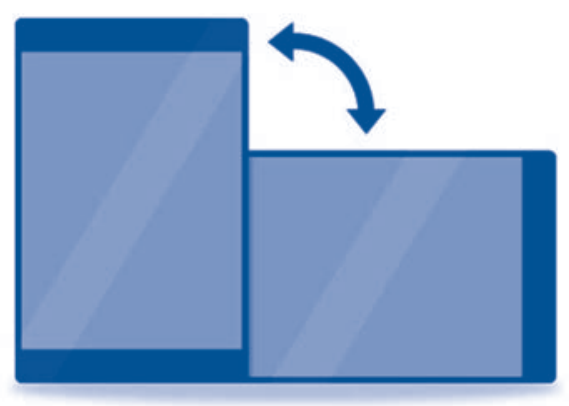

Figura 22. Orientación vertical y horizontal Fuente: Elaboración propia

Se desarrolla la aplicación móvil utilizando el sistema Android. A continuación se muestran las diferentes pantallas que tiene el sistema.

\section{Captura de pantallas}

Pantalla de Bienvenida

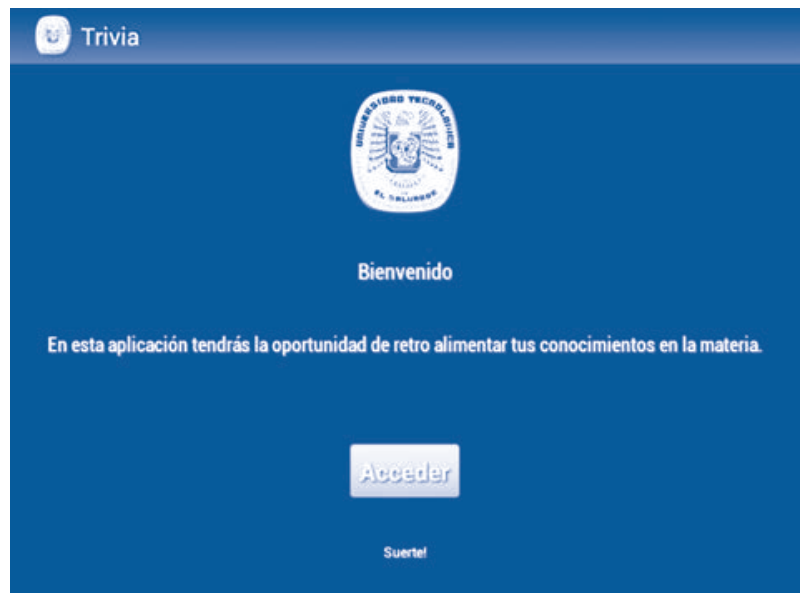

Figura 23. Pantalla de Bienvenida

\section{Retroalimentar}

En esta pantalla, el usuario tendrá que presionar el botón Acceder para entrar a la aplicación. 


\section{Presentación de niveles}

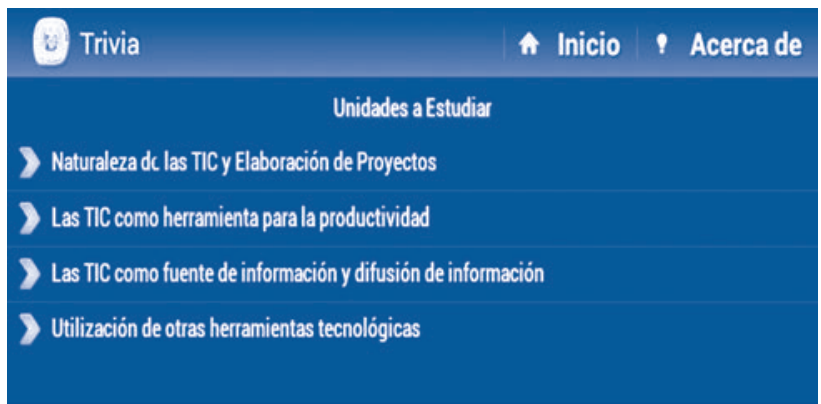

Figura 24. Presentación de niveles

Elaboración de proyectos

En esta pantalla aparecen las distintas unidades de la asignatura. El usuario debe seleccionar la unidad correspondiente. Cada unidad es dependiente de la anterior, es decir, funciona como si fueran niveles. La unidad 1 no tiene prerrequisitos; pero para acceder a las demás unidades, el usuario debe haber cumplido el número de puntos mínimos para avanzar a la siguiente unidad. Los puntos mínimos para pasar a otro nivel son 8.

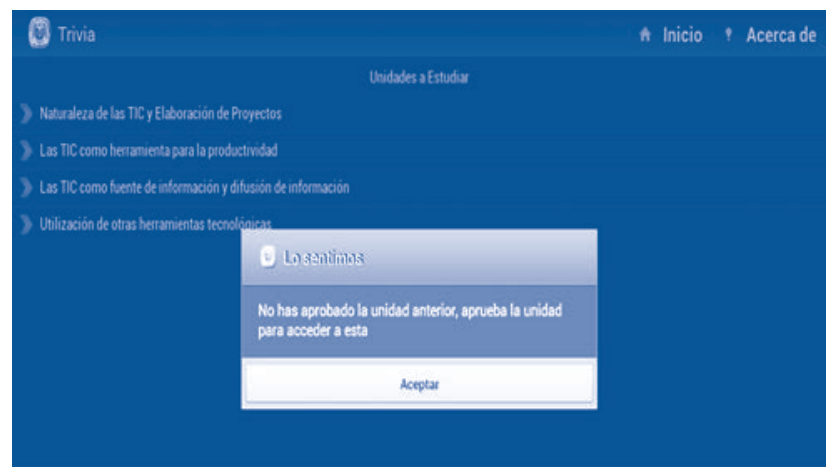

Figura 25.

Para avanzar a otro nivel debe pasarse el anterior

\section{Elaboración de proyectos}

A continuación se presentan capturas de pantallas con las preguntas que son parte del nivel uno de la trivia.

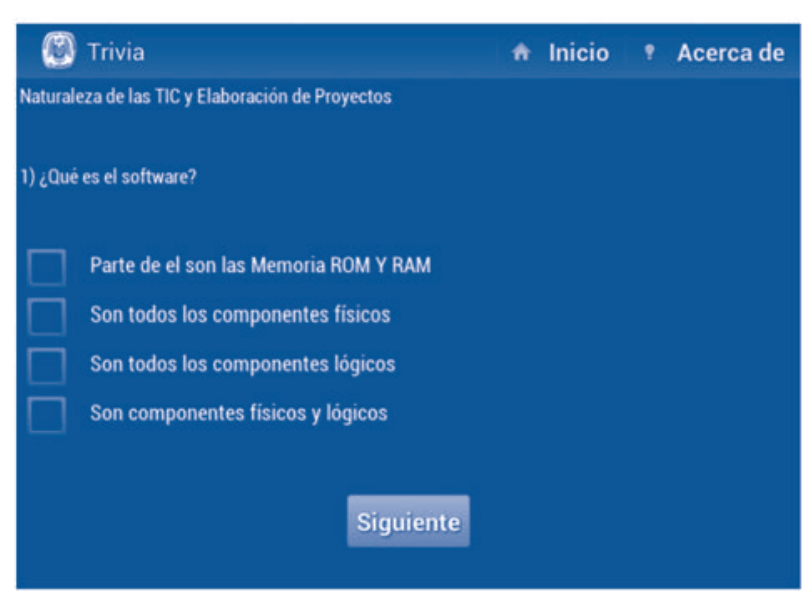

Figura 26.

Pantallas que muestran las 5 preguntas de la trivia que aparecen por cada nivel memoria
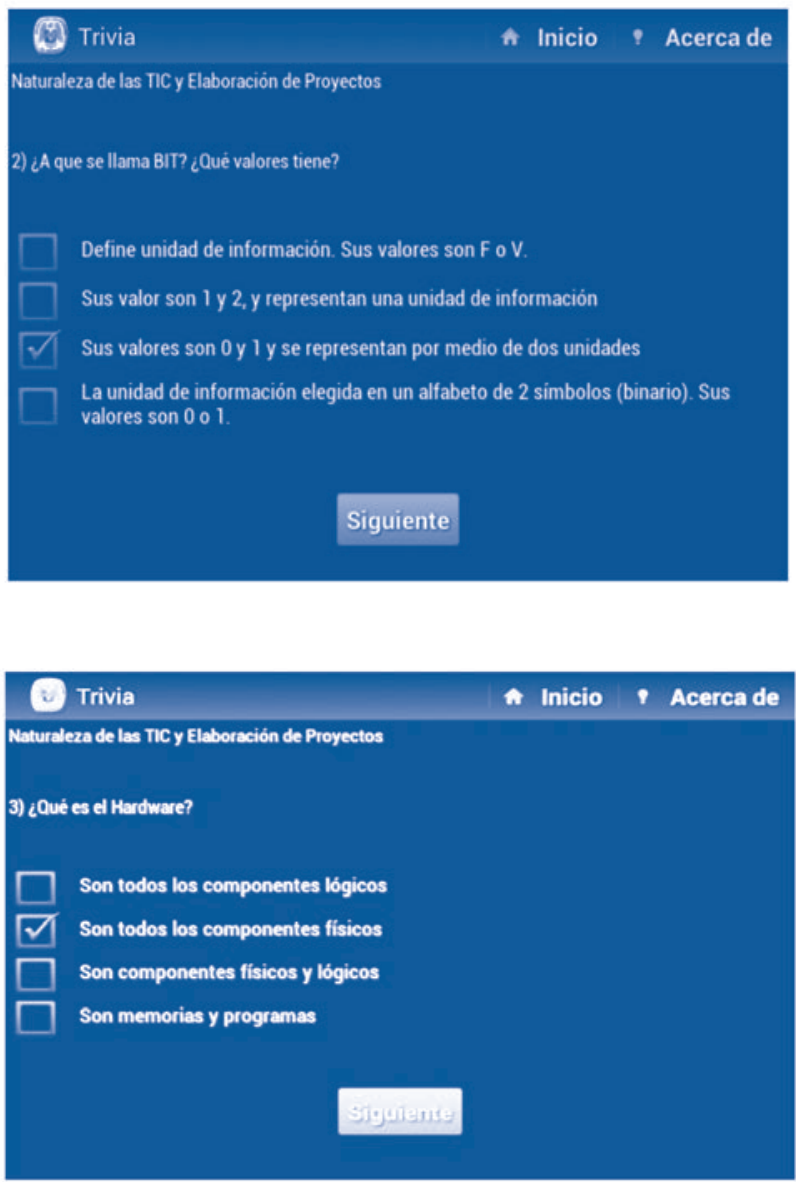

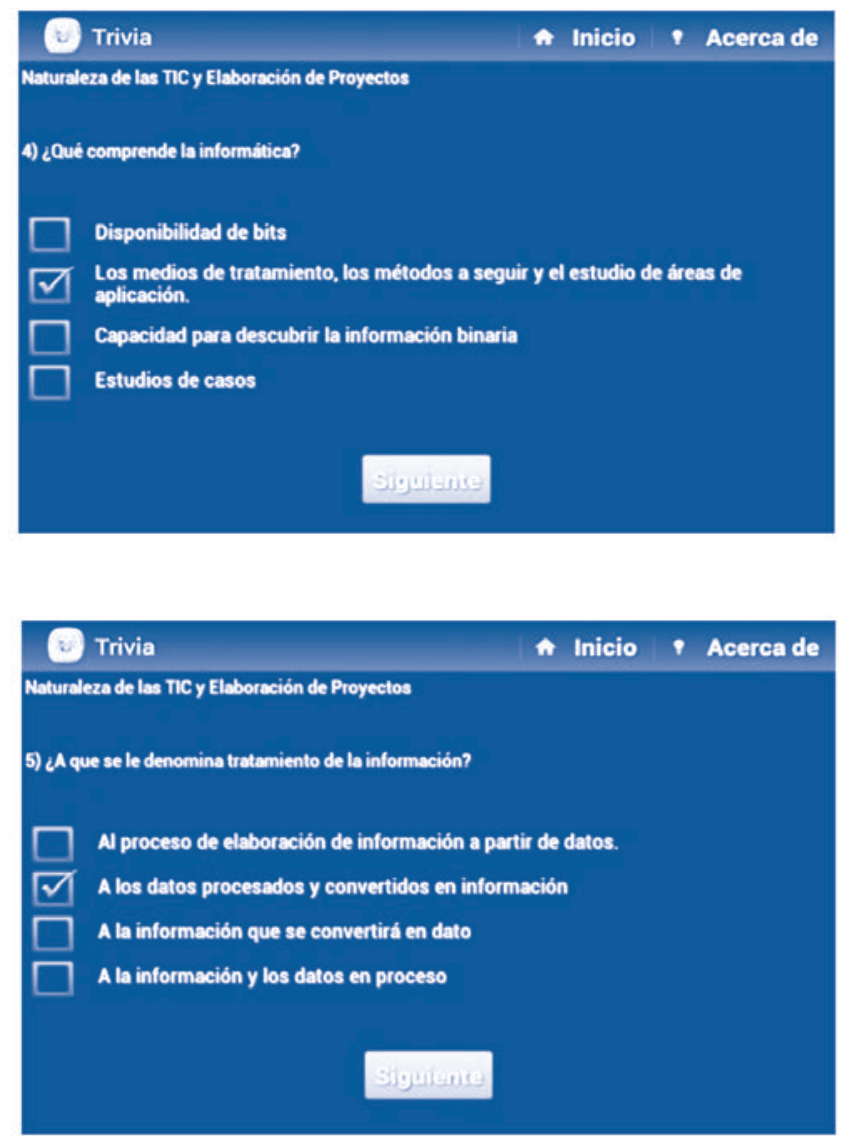

Al enviar la pregunta número 5 , inmediatamente el sistema realizará un resumen de la nota obtenida. Si fue acertada, felicitará al usuario y le permitirá avanzar al siguiente nivel. De lo contrario, le indicará las preguntas en las cuales falló, ubicándole un URL en donde están alojados los materiales bibliográficos que deberá leer para superar la prueba.

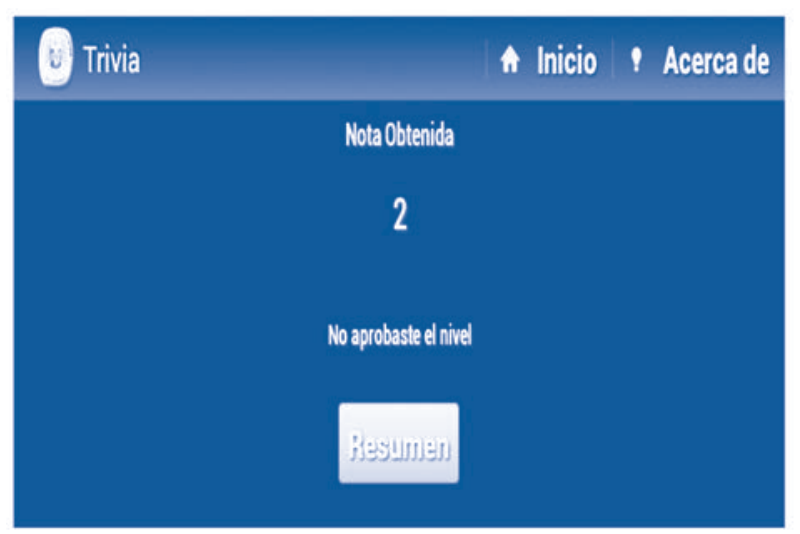

Figura 27.

Pantalla que muestra el resultado obtenido al realizar cada nivel

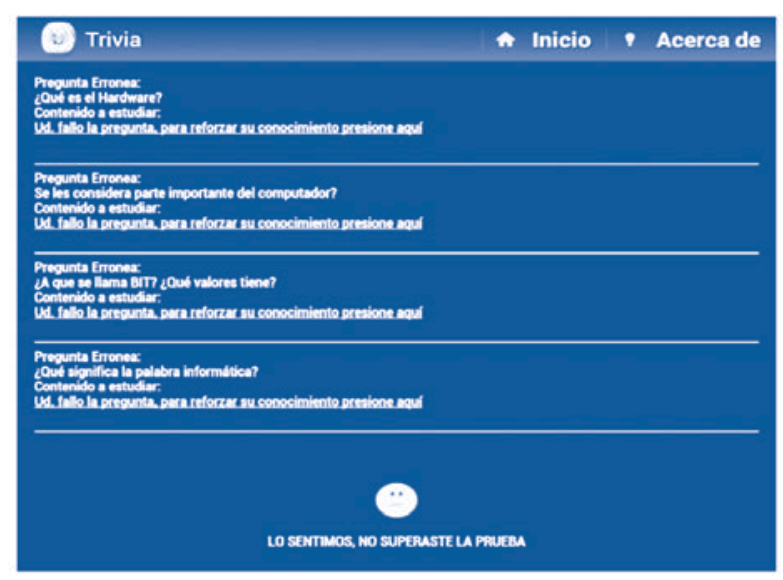

El usuario deberá estudiar el contenido relacionado con la pregunta que no respondió correctamente y después volver a iniciar la prueba.

Al aprobar el nivel, al usuario le aparecerá una pantalla como la siguiente:

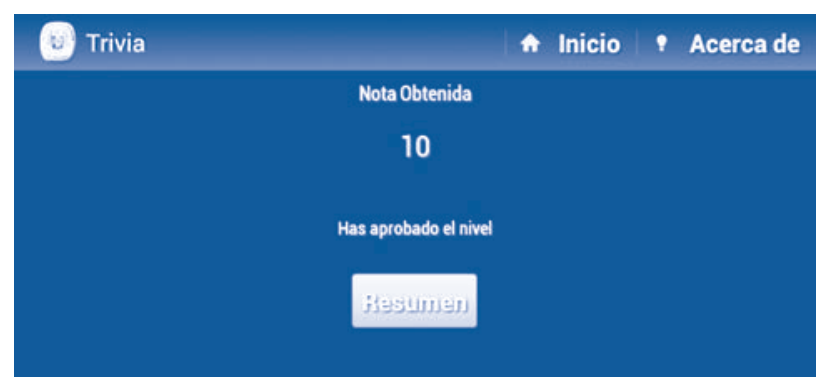

Figura 28.

Pantalla que aparece al aprobar un nivel obtenida

Ahora, el usuario de la aplicación puede continuar con las demás unidades. 


\section{Requisitos recomendados para instalar la aplicación}

El dispositivo móvil, para que pueda ejecutar la aplicación, debe cumplir con lo siguiente:

1. Sistema operativo Android versión 2.0 en adelante.

2. Procesador de $550 \mathrm{MHz}$.

3. Memoria RAM $256 \mathrm{MB}$.

4. Duración de la batería (mAh = miliamperio-hora).

5. Posibilidad de conexión a internet o intercambio de archivos por medio de Bluetooth o infrarrojo.

\section{Dispositivos móviles en los que se probó la aplicación}

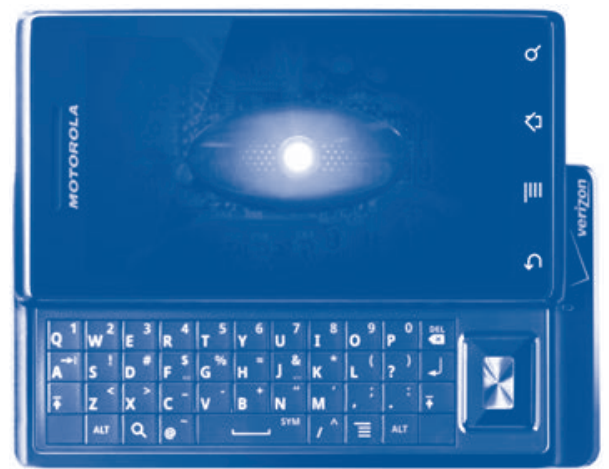

Figura 29. Motorola Verizon Wireles

Fuente: http://www.talkandroid.com/1824.verizon-motorola-droid

Motorola Verizon Wireles. Costo: 150 dólares. Características: Android 2.0, procesador $550 \mathrm{MHz}$, tamaño de pantalla 3.7 pulgadas, conexión a internet por medio de Wi-Fi, capacidad de la batería $1400 \mathrm{MHz}$.

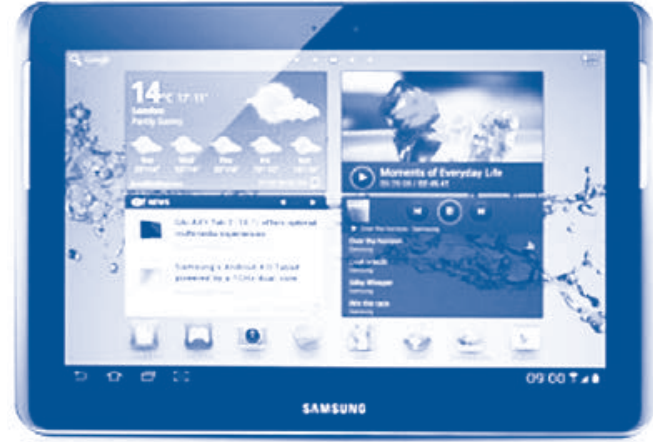

Figura 30. Samsung Galaxy Tab 2

Fuente: http://www.inode64.com

Samsung Galaxy Tab 2. Costo: 750 dólares. Características: Android versión 4.0, capacidad de la batería: 7.000 mAh, procesador $1,0 \mathrm{GHz}$, conexión a internet por medio de Wi-Fi, tamaño de pantalla 10.1 pulgadas.

\section{Dónde descargar la aplicación}

La aplicación puede ser descargada desde el URL: http:// tecno-logica.utec.edu.sv/aprende-movil/index.html.

Luego de bajar el archivo a su dispositivo móvil, deberá proceder a instalar la aplicación. Este proceso es muy fácil, por lo que rápidamente podrá comenzar a disfrutar de la trivia desde su dispositivo móvil.
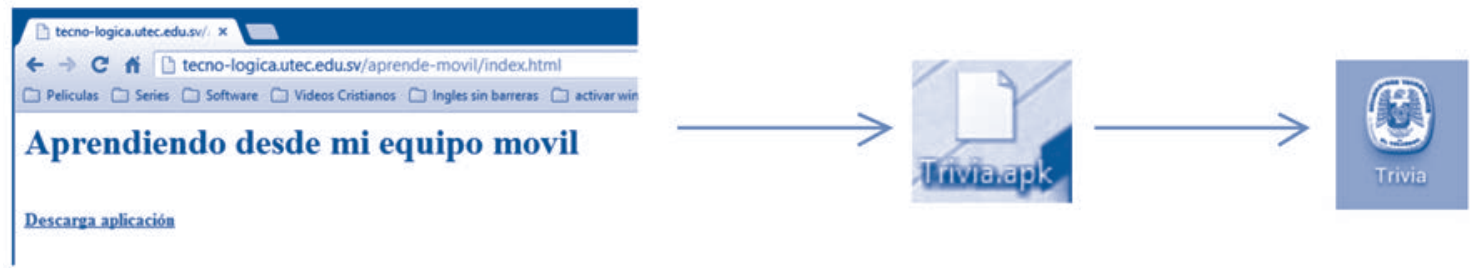

Figura 31. Pasos para descargar trivia

Fuente: Elaboración propia 


\section{Referencias}

Alonso, A.B. (S/N de S/N de 2011). Universidad de Oviedo. Obtenido deUniversidad de Oviedo: http://156.35.151 .9/ smi/5tm/09trabajos-sistemas/1/Memoria.pdf

Ambriz, M.L. (S/N de diciembre de 2011). Ilce. Obtenido de Instituto Latinoamericano de la Comunicación: http://ddd.uab.cat/pub/dim/dim_a2012m1n22/dim_ a2012m1n22a7.pdf

Arroyo, R. (16 de febrero de 2009). Itespresso. Recuperado el 15 de 11 de 2012, de Itespresso: http://www.itespresso.es/la-turbulenta-historia-de-palm-38897.html

Brain, L. (27 de 11 de 2011). EL CERN: Un logro europeo. Aula del memo, España. Obtenido de Sitio web de Wikipedia.

Caffa, A. (2008). Sistemas operativos para dispositivos móviles. S/N: S/N.

Cano, J.B. (S/N de S/N de 2007). Docencia.ac.upc.edu. Obtenido de Docencia.ac.upc.edu: http://docencia.ac.upc. edu/EPSC/PSE/documentos/Trabajos/Archivo/Trabajo_Linux_empotrado.pdf

Etxeberria, A.I. (2011). Mobile Learning: Aprendiendo Historia con mi teléfono, mi GPS y mi PDA. Madrid: S/N.

Gross, M. (26 de 10 de 2012). Android: Origen, evolución y liderazgo del sistema operativo para smartphones. Recuperado el 16 de 11 de 2012, de Android:
Origen, evolución y liderazgo del sistema operativo para smartphones: http://manuelgross.bligoo. com/20121026-android-origen-evolucion-y-liderazgodel-sistema-operativo-para-smartphones

Hernandez, E.F. (10 de 05 de 2011). Luxstevejobs. Recuperado el 15 de 11 de 2012, de luxstevejobs: http://luxstevejobs.comxa.com/obra1.html

Hernández, R. (12 de agosto de 2011). elearning.galileo. edu. Recuperado el 22 de 10 de 2012, de elearning. galileo.edu: http://elearning.galileo.edu/revista/index. php?option=com_content\&view=article\&id=20\&ltem id $=115$

López, L.M. (2010). El aprendizaje móvil (m-learning) como herramienta para el desarrollo del análisis critico en los alumnos de profesional. Mexico: S/N.

Mined (20 de 11 de 2012). Mined. Obtenido de Mined: http://www.mined.gob.sv/index.php/descargas. html?task=view.download \&cid=1474

Montoya, M.S. (12 de 2 de 2009). UTPL. Obtenido del sitio web UTPL: http://www.utpl.edu.ec/ried/images/pdfs/ vol12N2/recursostecnologicos.pdf

Rodríguez, B. (2012). Sistemas operativos para dispositivos móviles.

Tableta-Aérea. (29 de Octubre de 2010). Tableta aérea. Obtenido de Tableta aérea: http://www.tabletarea.com/ caracteristicas.html 